\title{
Nucleolus-organizing Chromosomes and Ribosomal Gene Amplification Phenomenon in Root Nodule Cells of Trifolium pratense: An Electronmicroscopic Study
}

\author{
Lucian Gavrila and Grigore Mihaescu \\ Genetics and Microbiology Departments, Bucharest University, \\ Aleea Portocalilor 1-3, sector 6, Bucuresti-77 206, Romania
}

Accepted August 8, 1991

The root nodules of legume plants are structures that represent a result of an abnormal, but harmonious, differentiated and limited tissue growth which is quantitatively different from that which takes place in other organs or in malignant tissues. The nodule shows a result of the interaction between root cells of the host plant and infecting nitrogen-fixing bacteria of the Rhizobium genus, leading to the most complex and perfect symbiotic association in which the plant offers its photosynthetic carbohydrates, while the bacteria, becoming bacteroids, use the energy that results from sugar degradation to reduce $\mathrm{N}_{2}$ to $\mathrm{NH}_{3}$.

In a previous paper (Mihaescu et al. 1985), we have analyzed the functional significance of cell structures from root nodules in Trifolium pratense. In the central zone of the nodule (core nodule), the main functional relationships between bacteroids and plant cells are performing. Immediately after infection, the root meristematic cells undergo endopolyploidization process (Caldwell and Vest 1977) and the bacteroids occupy the whole cytoplasmic space. Approximately 1000 bacteroids are encountered in a plant-host cell (Denarie and Truchet 1979). The endocycles which are preformed in the $T$. pratense host cells lead to endopolyploidization of these cells. As a consequence, their volume increases several times and, in such a way, the nodules are built. The situation is analogous to that encountered in gall formation on the leaves in Quercus, Vitis or Hedera in which the gallicicol insects are performing egg, larval and pupal stages of their life (Gavrila et al. paper in preparation).

There is also very close similarities between ribosomal gene amplification phenomenon which takes place in amphibian oocytes, particularly regarding the nucleolus desaggregation through which numerous extrachromosomal nucleoli result (Steopoe et al. 1985) and the nucleolus desaggregation in these root nodule cells, with the same result. These aspects suggest the conclusion that such root nodule cells are highly synthesizing cells which underwent a strict determination and narrow specialization processes, by sophisticated cytodifferentiation mechanisms, which may be of great interest for molecular biologists.

The permanent condensation of the nucleolus-organizing chromosome in the nuclei of the cells which constitute root nodules of $T$. pratense is comparable with the permanent condensation of polytenic chromosomes in cells of dipteran salivary glands or, even more properly with polytenic chromosomes from the cells of embryonic suspensor in Phaseolus coccineus (Nagl 1978), such chromosomes exhibiting a banding pattern, although irregular, atypical, but in some regions with very clear alternance of bands and interbands.

\section{Material and methods}

Efficient nodules were prefixed in $2 \%$ glutaraldehyde buffer and postfixed in $1 \%$ osmium tetraoxide. After dehydration, the pieces were embedded in Epon. The thin sections were stained with uranyl acetate and lead citrate and examied under JEM 7 and Philips 401 trans- 
mission electronmicroscopes.

\section{Results and discussions}

Root nodule cells of $T$. pratense exhibit peculiar features of nucleus and nucleolus structure (Figs. 1-3), evidentiated in electron microscopy. They lack heterochromatin and possess a permanently individualized nucleolus-organizing chromosome, being highly functionaly specialized cells, representing the link between two partners of this symbiotic association: bacteria of Rhizobium genus and the host plant $T$. pratense.

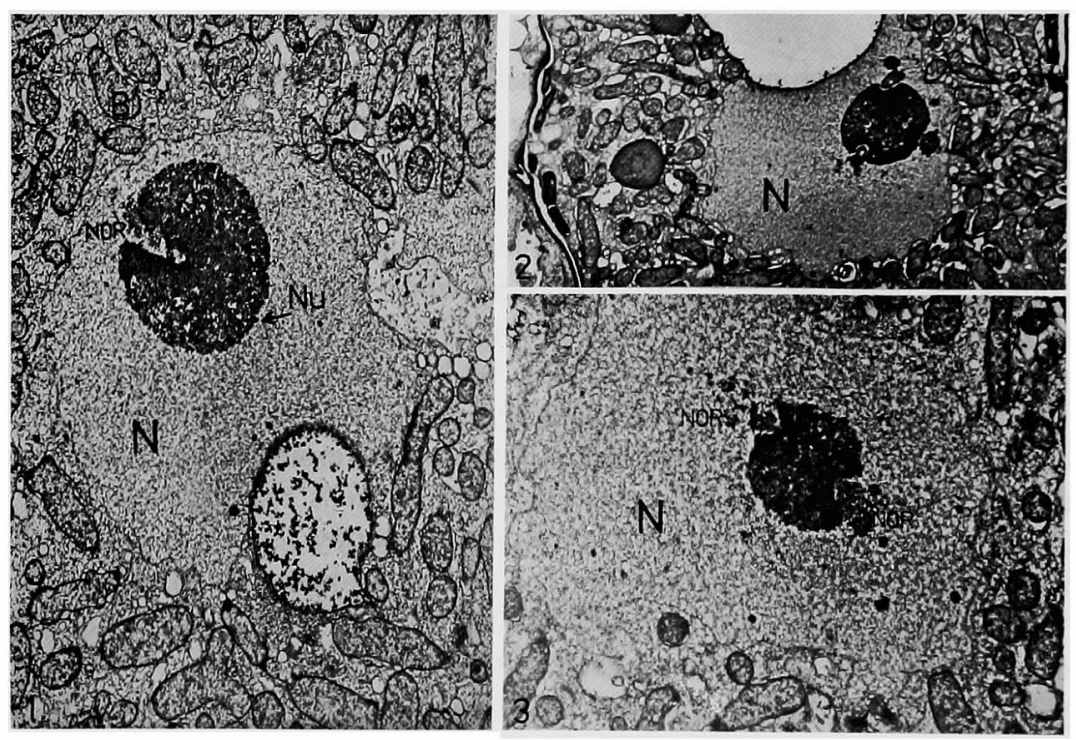

Figs. 1-3. The overall view of nucleus-cytoplasm interface in a root-nodule cell of Trifolium pratense in which the nucleus $(\mathrm{N})$, centrally located has an obvious nucleolus $(\mathrm{Nu})$ organized on one, two or even three nucleolar-organizing regions (NOR). The bacteroids (B), surrounded by fine peribacteroidal membrane have different shapes and size, $\mathrm{Y}$-shaped cells being characteristic. Note the uniform appearance of nuclear chromatin, homogenous and lacking heterochromatic blocks or condensed chromatin: $1, \times 7,500 ; 2, \times 5,000 ; 3, \times 6,000$

Our investigations revealed that these cells perform a ribosomal gene amplification phenomenon, the nucleolar-organizing region (NOR) of the nucleolus-organizing chromosome being not only transcribed at a high rate, but it is present in double or multiple copies on the same chromosome (Fig. 4), or even on different chromosomes as a consequence of initial polyploidization process which is performed by endocycles (Figs. 2, 3, 5, 6). In Fig. 4 the nucleolus-organizing chromosome is in fact a pair of homologous chromosomes in a somatic pairing process and as a consequence of polyteny such structure exbibits in some portions a banded pattern.

The decondensed state of nucleolar organizing region (NOR) facilitates the transcription process. In Fig. 4 the transcription units constituting such NORs are largely separated. This might mean that there is a repetitiveness of ribosomal cistrons located on the same chromosome in one nucleolar-organizing region as well as a repetitiveness of nucleolar-organizing regions on the same chromosome.

The nucleolus desaggregation is a high ordered process leading to the dispersion of the 
nucleolar material (i. e. of ribosomes) facilitating the ribosomes transfer into the cytoplasm. The process can be prefaced or accompanied by the release of nucleolar bodies of discoidalring shape from the large nucleoli as in Fig. 9 and especialy as in Fig. 10.

The absence of heterochromatic blocks from the nuclei of nodule cells could be interpreted in different ways, one of them being a selective endopolyploidization of functional euchromatin leading to amplification of the active genes, while the regulation of exchanges between hostcells and nitrogen-fixing bacteria (bacteroids) adapts other mechanism instead of those in which nuclear peripheral blocks of heterochromatin are involved. May be, the lack of such heterochromatic blocks facilitates a free exchange between these two partners of the symbiosis, the nodule cells which become veritable ammonia deposit and the bacteroids which are the producers.

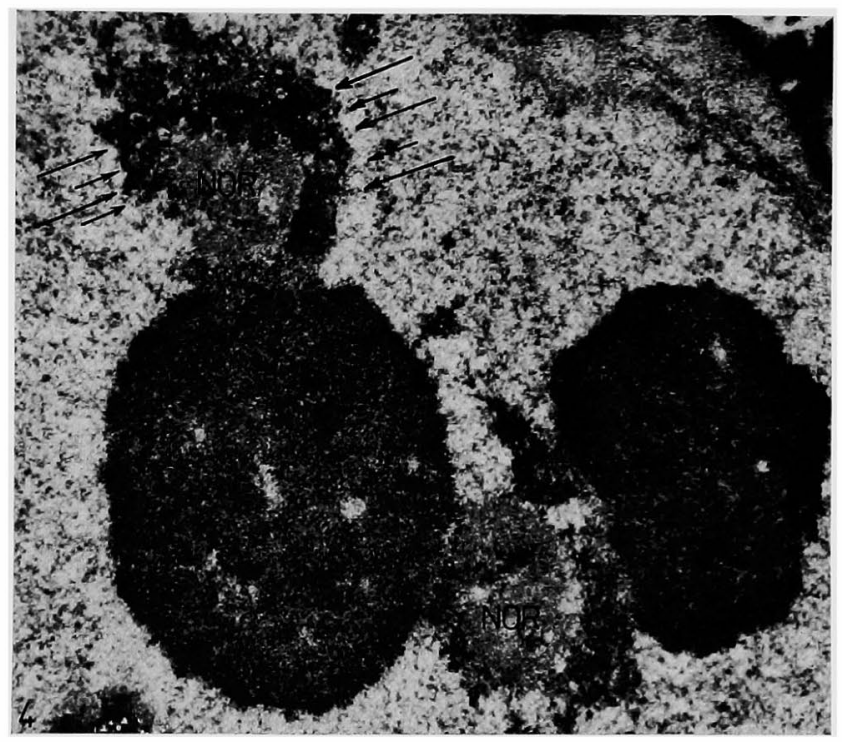

Fig. 4. A nucleolus-organizing chromosome with at least four nucleolar-organizing regions (NOR), two being at the end of their activity, building two large nucleoli, while other two are at the beginning of the process. The chromosome is in fact a pair of homologous chromosomes in a somatic pairing, exhibiting a model of bands and interbands, well expressed in the upper left end of this chromosome (long arrows indicate the bands, while short arrows indicate interbands); $\times 55,000$.

Which is the functional significance of such processes here, in the nodule cells? One can imagine the functional root-nodule as a miniature "ammonia factory". It receives the carbohydrates through vascular system of the host plant to be used by bacteroids to obtain the necessary energy for the nitrogen reduction. The resulted combined nitrogen is transferred into the vascular system of the host plant. An efficient function at high parameters of such "ammonia factory" implies a differential functioning of the genes: the ribosomal genes, which direct protein synthesis are amplified, while the other genes could be kept at diploid level or even eliminated if they are not essential and are located in heterochromatic regions of the chromosomes. In the nuclei of nodule cells there are not usual peripheral heterochromatic blocks encountered in all eukaryotic cells, being in intimate contact with the inner membrane of nuclear envelope. As a result of endopoliploidization process of the root cortex cells, at the beginning of infectior with Rhizobium, all the genes could be amplified or, at least, most of 
them. Later, as the infection process is proceeding and the symbiosis with the root cells is well established, some genes are inactivated by heterochromatinization process. Eventually, such genes are destroyed by elimination of heteochromatic blocks from nucleus into the cytoplasm. Alternatively, the specific amplification of ribosomal genes can be achieved by an extrareplication of ribosomal cistrons through polytenization, such ribosomal cistrons being localized in nucleolar-organizing region of the nucleolus-organizing chromosome, and it is the only chromosome which remain condensed in the interphase period as well as it is the chromosome which exhibit banding pattern. The nodule cells are, at the beginning of their dif-
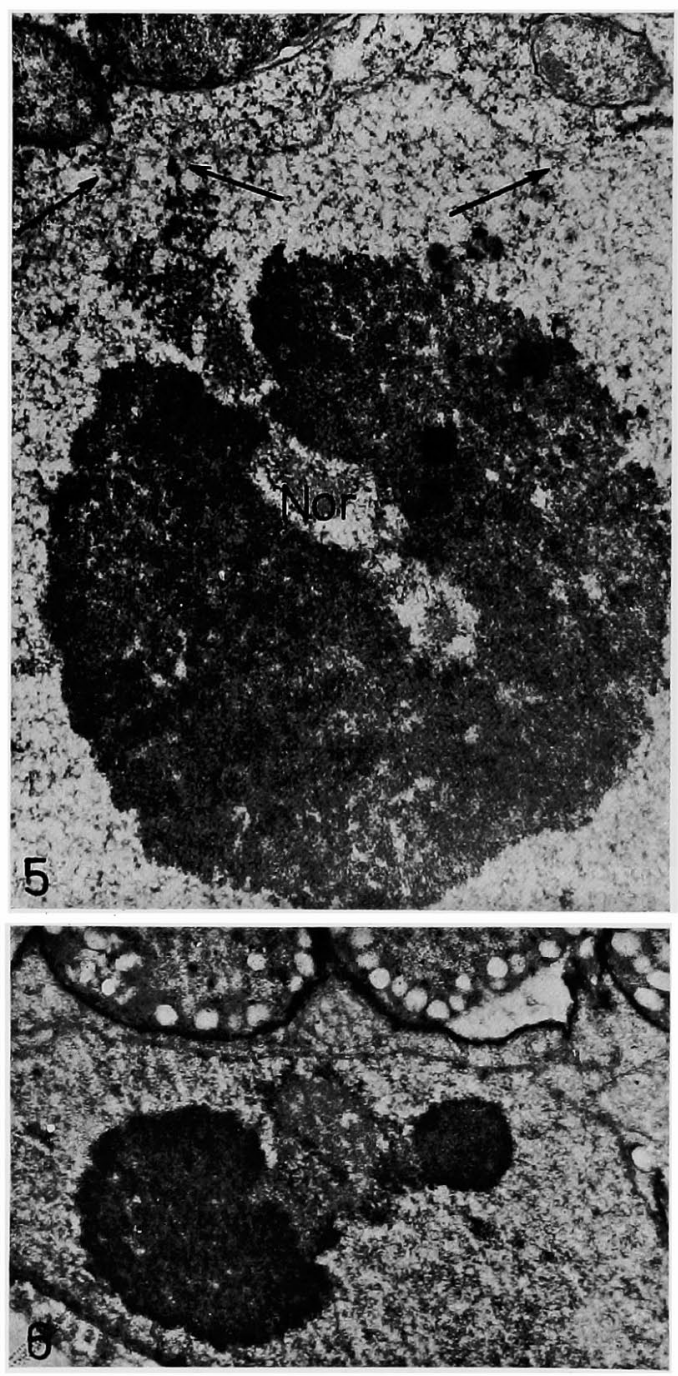

Figs. 5, 6. The nucleolus-organizing chromosomes attached at internal membrane of nuclear envelope (arrows) appear as double, condensed, fibrous structure, each having its point of attachment, suggesting a somatic chromosomal pairing of two homologous nucleolus-organizing chromosomes, each belonging to a haploid set of chromosomes in a diploid cell, before endoreduplication process started. In Fig. 5 three NORs (two being on the synapsed chromosomes, the other one being on a separate chromosome) are organizing the same nucleolus, while in Fig. 6 each NOR of the synapsed chromosomes organizes separately a nucleolus. Note in Fig. 6 the clear vacuoles at the periphery of bacteroids: $5, \times 37,000 ; 6, \times 15,000$. 
ferentiation highly active in the leghemoglobin synthesis, which is a sine qua non condition for the nitrogenase function, this substance fixing the free oxygen and creating an anoxic cellular environment necessary for the nitrogenase to be active in nitrogen fixation. These cells addopts polyteny as a strategy for ribosomal gene amplification, this superproduction of ribosomes being requested for a high leghemoglobin production in a short period of time.
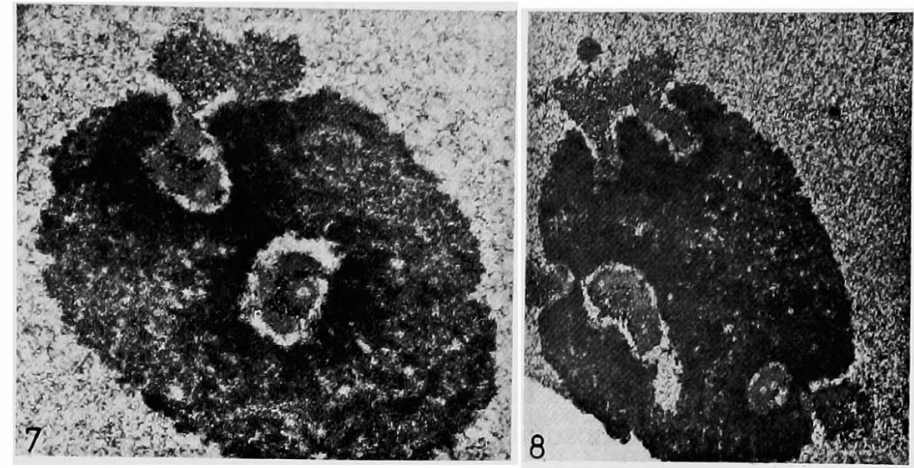

Figs. 7, 8. Multiple nucleolus-organizing chromosomes involved in the building of the same nucleolus of large size in a root-nodule endopolyploid cell: $7, \times 30,000 ; 8, \times 25,000$.

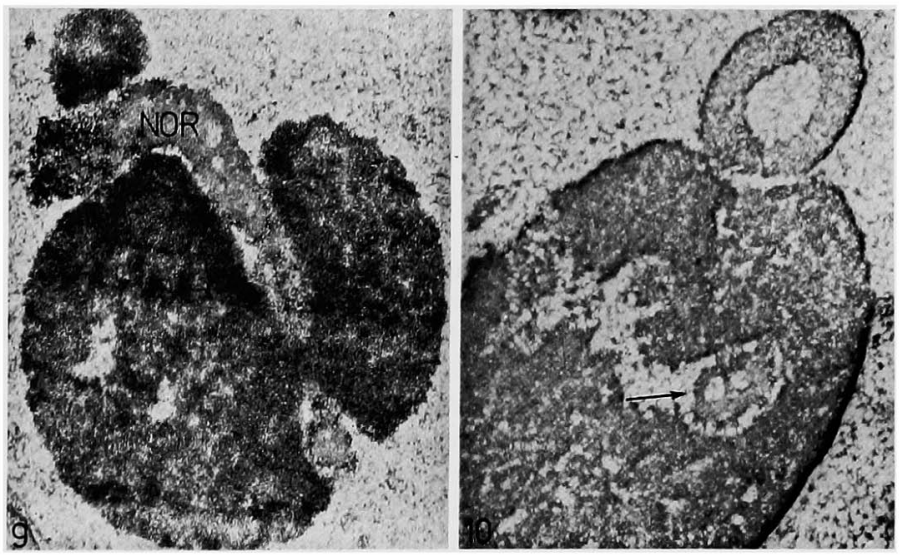

Figs. 9, 10. A longitudinal section through a nucleolar-organizing region (NOR) evidentiating its partial decondensed state compared with the end of nucleolus-organizing chromosome which is more condensed (upper left side). At the same time, the nucleolus desaggregation is obvious leading to the formation of three separate nucleolar bodies (Fig. 9) In Fig. 9, a cross-sectioning through an extremely decondensed and consequently highly transcribed nucleolar-organizing region (NOR) is presented. The release of a nucleolar body of discoidal-ring shape is evidentiated in the upper right side of Fig. 10. Another similar nucleolar body is inside of this desaggregating nucleolus (arrow); $9, \times 20,000 ; 10, \times 30,000$.

The hypertrophy of a single or of multiple nucleoli signifies the existence of an intense ribosomal genes transcription, followed by a rapid assemblage of ribosomal subunits, necessary for protein synthesis from nodule cells, such as leghemoglobin and the enzymes which perform the coupling of ammonia excreted by bacteroids with carbon chains from which amino acids are synthesizing (Beneman and Valentine 1972, Brill 1976). The total absence of heterochromatin can be interpreted as facilitating nucleo-cytoplasmic exchanges in the cells of the functional nodule, such cells being high functionally specialized units to serve as intermediary step 
in the symbiosis between bacteroids and the host plant.

$\mathrm{Nagl}$ (1982) has pointed out that the nuclei with diffuse euchromatin always possess heterochromatin in the form of chromocenters, so that this type of nuclei is known as "chromocenter type". Nuclei with chromomeric and chromonematic euchromatin organization may also possess chromocenters in addition to the patches or strands of condensed euchromatin.

In Trifolium pratense root-nodule cells we have not found any one of these three nuclear types generaly encountered in plants. Here, we are facing with a special case of nuclearstructural/functional organization, structural complexity being positively correlated with increasing 2C DNA content of the nodule cells as Nagl and Fusenig (1979, cited by Nagl, 1982) pointed out for polyploid species.

\section{Summary}

The nucleus of root nodule cells of Trifolium pratense exhibits peculiar features which has been investigated by TEM. It lacks heterochromatin and possesses a penmanently individualized nucleolus-organizing chromosome. These cells perform a ribosomal gene amplification phenomenon, the nucleolar-organizing-region of the nucleolus-organizing chromosome being not only transcribed at a high rate, but it is present in double or multiple copies on the same chromosome, or on different chromosomes as a consequence of initial polyploidization process perfomed by endocycles. This process is later followed by polytenization of nucleolusorganizing chromosomes which, as a consequence, exhibit a discrete or, in some regions, an obvious banded pattern. Such genetic processes are not unexpected in these highly functionally specialized cells which are parts of the miniature "ammonia factory" represented by a functional rootnodule.

\section{References}

Beneman, J. R. and Valentine, R. C. 1972. The pathways of Nitrogen Fixation. Advances in Microbial Physiology. Ed. Rose A. H. et Tempest. D. W. Acad. Press, 8, 59-104.

Brill, W. J. 1976. Biological Nitrogen Fixation. Sci. Amer. 236, 3, 68-81.

Caldwell, B. E. and Vest, H. G. 1977. Genetic aspects of nodulation and nitrogen fixation by Legumes. The Macrosymbiont. In: Treatise on Dinitrogen Fixation. eds Hardy, R. W. F. and Sylver, W. S., J. Wiley \& Sons, New York, London, Toronto, Sydney.

Denarie, J. and Truchet, G. 1979. La symbiose Rhizobium Legumineuses: rôles respectifs des partenaires. Physiol. Vég. 17, 643-667.

Mihaescu, Gr. Gavrila, L. and Georgescu, D. 1985. Functional significance of cell structures from root nodules in Trifolium pratense. Rev. Roum. Biol. Biol. veget. 30, 169-173.

Nagl, W. 1978. Endopolyploidy and Polyteny in Differentiation and Evolution. North Holland, Amsterdam.

Nagl, W. 1982. Condensed Chromatin: species-specificity, tissue-specificity and cell-cycle specificity, as monitored by scanning cytometry. in: Cell Growth ed. C. Nicolini, Plenum Press, New York and London.

Steopoe, I. L. Gavrila, Gr. Mihaescu and D. Comsa, 1985. The study of lampbrush chromosomes in relation with ribosomal gene amplification in oocytes of Triturus cristatus and Salamandra salamandra. Anal. Univ. Buc. XXXIV, 3-9. 